\title{
Cervical cancer incidence after normal cytological sample in routine screening using SurePath, ThinPrep, and conventional cytology: population based study
}

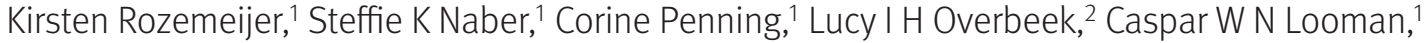 \\ Inge M C M de Kok, ${ }^{1}$ Suzette M Matthijsse, ${ }^{1}$ Matejka Rebolj, ${ }^{3}$ Folkert J van Kemenade, ${ }^{4}$ \\ Marjolein van Ballegooijen ${ }^{1}$
}

\section{ABSTRACT}

OBJECTIVE

To compare the cumulative incidence of cervical cancer diagnosed within 72 months after a normal screening sample between conventional cytology and liquid based cytology tests SurePath and ThinPrep.

DESIGN

Retrospective population based cohort study.

SETTING

Nationwide network and registry of histo- and cytopathology in the Netherlands (PALGA), January 2000 to March 2013.

POPULATION

Women with 5924474 normal screening samples (23833123 person years).

\section{EXPOSURE}

Use of SurePath or ThinPrep versus conventional cytology as screening test.

\section{MAIN OUTCOME MEASURE}

72 month cumulative incidence of invasive cervical cancer after a normal screening sample for each screening test. Cox regression analyses assessed the hazard ratios, adjusted for calendar time, age, screening history, and socioeconomic status and including laboratories as random effects.

RESULTS

The 72 month cumulative cancer incidence was 58.5 ( $95 \%$ confidence interval 54.6 to 62.7) per 100000 normal conventional cytology samples, compared with

\section{WHAT IS ALREADY KNOWN ON THIS TOPIC}

The general consensus is that sensitivity for cervical intraepithelial neoplasia (CIN) $\mathrm{II}^{+}$is similar between liquid based cytology and conventional cytology

However, recent studies have shown that sensitivity for detecting CIN II+ lesions depends on the type of liquid based cytology test used (SurePath or ThinPrep)

Whether differences exist in the sensitivity for progressive $\mathrm{CIN} \mathrm{II}^{+}$lesions between SurePath, ThinPrep, and conventional cytology is unknown

\section{WHAT THIS STUDY ADDS}

Using SurePath rather than conventional cytology or ThinPrep as the primary test method was associated with lower cervical cancer rates diagnosed after a normal screening sample

This strongly suggests that the sensitivity of SurePath to detect progressive CIN lesions is higher

Using ThinPrep rather than conventional cytology as the primary test method seemed to be associated with higher cervical cancer rates diagnosed after a normal screening sample, although differences were non-significant

66.8 (56.7 to 78.7) for ThinPrep and 44.6 (37.8 to 52.6) for SurePath. Compared with conventional cytology, the hazard of invasive cancer was 19\% lower (hazard ratio $0.81,95 \%$ confidence interval 0.66 to 0.99 ) for SurePath, mainly caused by a $27 \%$ lower hazard $(0.73$, 0.57 to 0.93 ) of a clinically detected cancer. For ThinPrep, the hazard was on average $15 \%$ higher (hazard ratio 1.15, 0.95 to 1.38), mainly caused by a $56 \%$ higher hazard of a screen detected cancer (1.56, 1.17 to 2.08 ).

\section{CONCLUSIONS}

These findings should provoke reconsideration of the assumed similarity in sensitivity to detect progressive cervical intraepithelial neoplasia between different types of liquid based cytology and conventional cytology.

\section{Introduction}

The use of conventional cytology as the primary test method has been replaced by the use of liquid based cytology in many countries with organised cervical cancer screening programmes, such as the UK, the Netherlands, and Denmark. ${ }^{12}$ The main advantages of using liquid based cytology instead of primary conventional cytology are facilitation of reflex testing (that is, the residual material can be tested for the presence of the human papillomavirus in case of borderline/mildly dyskaryotic smears) ${ }^{34}$ and reduction in the number of slides of unsatisfactory quality. ${ }^{5-9}$ The sensitivity of liquid based cytology for detecting cervical intraepithelial neoplasia (CIN) $\mathrm{II}^{+}$lesions is believed to be similar to that of conventional cytology. ${ }^{10}{ }^{11}$ However, the literature has been dominated by many studies comparing CIN detection between ThinPrep and conventional cytology, ${ }^{12-17}$ whereas only two studies have compared CIN detection between SurePath and conventional cytology. ${ }^{718}$ Therefore, we compared CIN $\mathrm{II}^{+}$detection rates between these three types of cytology tests in our previous study, including more than six million smears taken within the Dutch cervical cancer screening programme. ${ }^{19}$ Whereas the use of SurePath led to an $8 \%$ increase in detection of $\mathrm{CIN} \mathrm{II}^{+}$compared with conventional cytology, the use of ThinPrep did not affect CIN $\mathrm{II}^{+}$detection rates. These results were compatible with the results of other studies. ${ }^{12-1620}$

Detecting more CIN as a result of an abnormal screening test is expected to deplete the pool of lesions that would have progressed to cancer. However, in the absence of screening (and associated treatment) only a fraction of screen detected CIN would progress to 
cervical cancer (that is, the progressive CIN lesions), so detecting more CIN lesions is not always equivalent to preventing more cervical cancers. To assess whether the ability to detect progressive CIN lesions differs between the types of screening tests, the probability of a diagnosis of invasive cervical cancer in the period after a normal test result (the progressive CIN that screening has missed) has to be compared. As the incidence of invasive cervical cancer after a normal test result is low (six year cumulative incidence rate 48 (95\% confidence interval 43 to 54) per 100000 normal smears), ${ }^{21}$ such a comparison can be made only by using an observational population based study in which a large number of samples can be evaluated.

In the Netherlands, organised cervical cancer screening has existed since the 1980s, and women aged 30 to 60 years have been invited every five years since 1996. Until 2016, the screening strategy consisted of primary cytology screening with cytology triage testing, the latter either alone or in combination with human papillomavirus testing. All cervix uteri cytological and histological tests taken inside and outside the Dutch screening programme are registered in the nationwide network and registry of histo- and cytopathology in the Netherlands (PALGA). ${ }^{22}$ By using these data, we assessed the cumulative incidence of invasive cervical cancer detected within 72 months after a normal screening test result (diagnosed in the next screening round or outside the organised Dutch screening programme). If this incidence varies among the three tests (SurePath, ThinPrep, and conventional cytology), the sensitivity for progressive CIN lesions probably differs between them. In addition, we stratified for the reason for cervical examination that led to the detection of cervical cancer (screen detected when programme smear detected or clinically detected in all other cases, which includes opportunistic screening as well as direct biopsies).

\section{Methods}

Information on all cytological and histological examinations of the cervix uteri taken in the Netherlands between January 2000 and March 2013 were available and retrieved from the national PALGA database. Multiple quality checks ensured the reliability of the retrieved data. ${ }^{2324}$ We identified women through their birth date and the first eight letters of their (maiden) family name. This identification code enables linkage of multiple tests belonging to the same woman, allowing us to follow individual screening histories.

We identified and selected episodes starting with a normal primary screening sample taken within the Dutch screening programme between January 2000 and March 2012. We identified screening samples through the reason for taking the sample being participation in the programme, which is routinely registered in PALGA. We also selected women with a primary sample of inadequate quality followed by a normal sample within the same episode. We defined an episode as starting with a primary test followed by one or more secondary tests in case the result was abnormal (at least borderline or mild dyskaryosis) or of inadequate quality. Unless the follow-up of a primary test had already been completed according to the guidelines (for example, by two consecutive normal samples after a screening result with borderline dyskaryosis), we considered tests taken within four years after a primary test to be secondary tests. ${ }^{25}$ We labelled all other tests as primary tests.

We stratified normal primary screening samples by the type of cytology test used (SurePath, ThinPrep, or conventional cytology). As PALGA does not register this routinely, regional coordinating pathologists obtained conversion dates (fixed to the first date of the yearly quarter) from the individual laboratories located in their region. In the Netherlands, it is standard practice for laboratories to supply general practitioners with cytology kits and thereby determine the type of cytology test that is used. We linked these conversion dates to the laboratory involved and the examination date as a proxy for which type of primary cytology test was used.

Follow-up ended at the date of the primary test of the next episode, which resulted in a cervical cancer diagnosis (a case) or not, on 31 March 2013 (the end of the database), or on completion of the 72 month period, whichever came first. We censored the follow-up at 72 months because it covers the invitation for the next screening round, which takes place 60 months after a normal screening sample. By definition, after a normal primary screening test, all new tests give rise to a new episode regardless of the reason for taking the test and the type of test. We identified histologically confirmed cases of cervical cancer by selecting all PALGA records that included pathology codes describing invasive cancers originating in the cervix uteri. These codes were manually checked to avoid over-counting of both non-invasive lesions and primary cancers originating elsewhere.

As women in the Netherlands are invited for screening in the year they turn 30, 35, 40, 45, 50, 55, and 60, we categorised age as 29-33, 34-38, 39-43, 44-48, 49-53, 54-58, and 59-63 years at the time of the normal primary cytological sample. We also defined calendar year at the time of the normal cytological sample. We defined socioeconomic status, categorised as low, middle, or high, by the status score. This is an ecological variable based on the household characteristics of the four digit postcode area where the woman was living at the time of the primary test. ${ }^{26}$ Status scores per four digit postal code came from the Netherlands Institute for Social Research and were based on mean income, percentage of households with a low income, percentage of households with (on average) a low education, and unemployment rate in 2010. Low socioeconomic status corresponded to a status score lower than -1 (that is, average status score minus 1 standard deviation), intermediate socioeconomic status to a score of between -1 and 1, and high socioeconomic status to a score higher than 1 (that is, average status score plus 1 standard deviation). We categorised screening history as no history of cytological smears (inside or outside the screening programme) before the normal screening sample, one cytological smear that was taken less than seven years before the normal screening sample, one cytological 
smear that was taken more than seven years before the normal screening sample, at least two cytological smears with the last being taken less than seven years before the normal screening sample, and at least two cytological smears with the last being taken more than seven years before the normal screening sample.

\section{Statistical analyses}

Laboratories implemented liquid based cytology testing at different points in time. Therefore, we expected follow-up and calendar time to differ between the three types of cytology tests. As demographic characteristics of screened women (age, screening history, and socioeconomic status) probably differed between laboratories, we expected them to differ between the types of cytology tests as well. As age, socioeconomic status, screening history, and calendar time were all associated with CIN and/or cervical cancer detection rates, ${ }^{27-30}$ they were all potential confounding factors. We used a Pearson's $\chi^{2}$ test to test whether their distributions differed between the types of cytological tests. We considered a $P$ value of less than 0.05 to be statistically significant. We imputed missing values with 10 imputation sets.

\section{Cumulative incidence and hazard ratio}

For each type of cytology test, we did a Kaplan-Meier analysis to calculate the cumulative incidence of invasive cervical cancer per 100000 normal cytological screening samples. We took differences in follow-up time into account and estimated the 95\% confidence intervals by non-parametric Kaplan-Meier product-limit estimator for $\log$ (hazard). ${ }^{2131}$ We used the R package "coxme" to do multilevel Cox regression analyses to compare the hazard of cervical cancer between the types of cytology tests, taking differences in follow-up time into account and adjusting for the confounding factors calendar time, socioeconomic status, age, and screening history. We included the determinant laboratory as a random effect in the model to take account of clustering of the data at the laboratory level. In addition, we stratified for the reason for the cervical examination that led to the cervical cancer diagnosis (that is, screen detected when detected by a programmed smear or clinically detected in all other cases, including opportunistic screening as well as direct biopsies). We tested time dependencies of the hazard ratios statistically by splitting the total follow-up time into two periods with a roughly equal number of cases. Subsequently, we assessed hazard ratios for each time period. If the sum of the deviance of both sub-models was significantly lower than the deviance of the original model, we considered the hazard ratio to be time dependent as it differed significantly between the time periods.

\section{Sensitivity analyses}

In the first sensitivity analysis, we restricted our Cox regression analysis to women with at least one previous smear. In the subsequent sensitivity analysis, we selected only women who attended the next screening round (within six years after a normal screening test result) in order to examine the effect that possible differences in the attendance rates at the next screening might have had on the comparisons between the cytology tests. We repeated the latter analysis in the third sensitivity analysis with the addition of an extra confounding factor, the type of cytology test used in the subsequent screening round. We did this to correct for the potential differences in the sensitivity of the subsequent screening test.

\section{Difference in CIN detection rates per 100000} screening samples and 72 month cumulative cervical cancer incidence after 100000 normal screening samples

We assessed the difference in CIN detection rates per 100000 SurePath and 100000 ThinPrep samples (compared with the CIN detection rates per 100000 conventional cytology samples) and compared it with the difference in the 72 month cumulative cervical cancer incidence after 100000 SurePath and ThinPrep normal screening samples. Information on the calculation of the difference in detection rates per 100000 primary screening samples can be found in the supplementary material. We calculated the 72 month cumulative cancer incidence rates for SurePath and ThinPrep by multiplying the distribution of the 72 month cumulative cancer incidence rate for conventional cytology with the distribution of the adjusted hazard ratios for SurePath and ThinPrep versus conventional cytology, as obtained by Cox regression.

\section{Patient involvement}

No patients or women eligible for screening were involved in the design and conduct of the study, nor were they involved in defining the research question or outcome measures. We have no intentions to disseminate our results to patients or women eligible for screening.

\section{Results}

Within the follow-up period, 1042 invasive cervical cancers were diagnosed after 3028865 normal conventional cytology samples, 231 cancers were diagnosed after 1303817 normal SurePath samples, and 328 cancers were diagnosed after 1591792 normal ThinPrep samples (table 1). This corresponds to 7.6, 4.8, and 6.3 cervical cancer diagnoses per 100000 person years, respectively.

\section{Crude cumulative incidence}

Compared with conventional cytology, the 12, 24, 36, 48, 60 , and 72 month cumulative incidences of invasive cervical cancer were significantly lower for SurePath (fig 1). When we compared SurePath with ThinPrep, all but the 24 month cumulative incidences were significantly lower for SurePath. No significant differences were apparent between ThinPrep and conventional cytology. The 72 month cumulative incidence was 44.6 (95\% confidence interval 37.8 to 52.6) after 100000 normal SurePath samples, 58.5 (54.6 to 62.7) after 100000 normal conventional cytology samples, and 66.8 (56.7 to 78.7) after 100000 normal ThinPrep samples. 


\begin{tabular}{|c|c|c|c|c|}
\hline Characteristics & Conventional & SurePath & ThinPrep & P value \\
\hline Normal screening samples & 3028865 & 1303817 & 1591792 & \\
\hline Person years at risk & 13796018 & 4835917 & 5201188 & \\
\hline Normal screening sample followed by subsequent screening ${ }^{*}$ & $1931397(63.8)$ & $445726(34.2)$ & $370519(23.3)$ & $<0.001$ \\
\hline Median (interquartile range) normal screening samples per woman & $1(1-2)$ & $1(1-1)$ & $1(1-1)$ & $<0.001$ \\
\hline Invasive cervical cancers diagnosed after normal screening sample & 1042 & 231 & 328 & $<0.001$ \\
\hline Screen detected $^{\dagger}$ & 414 & 84 & 103 & $<0.001$ \\
\hline Clinically detected $\neq$ & 628 & 147 & 225 & $<0.001$ \\
\hline \multicolumn{4}{|l|}{ Follow-up time: } & \multirow{7}{*}{$<0.001$} \\
\hline 0-12 months & 208668 (6.9) & 73905 (5.7) & $95563(6.0)$ & \\
\hline 12-24 months & $105945(3.5)$ & $191027(14.7)$ & $321784(20.2)$ & \\
\hline 24-36 months & $129165(4.3)$ & $187410(14.4)$ & 311295 (19.6) & \\
\hline 36-48 months & $203768(6.7)$ & $189063(14.5)$ & $284262(17.9)$ & \\
\hline 48-60 months & $920825(30.4)$ & $334677(25.7)$ & $339590(21.3)$ & \\
\hline 60-72 months, & $1460494(48.2)$ & $327735(25.1)$ & $239298(15.0)$ & \\
\hline \multicolumn{4}{|l|}{ Age, years: } & \multirow{8}{*}{$<0.001$} \\
\hline $29-33$ & $411873(13.6)$ & $167015(12.8)$ & $193998(12.2)$ & \\
\hline $34-38$ & $503889(16.6)$ & $187179(14.4)$ & $217213(13.6)$ & \\
\hline $39-43$ & $516728(17.1)$ & $218559(16.8)$ & $267194(16.8)$ & \\
\hline $44-48$ & $482822(15.9)$ & $218476(16.8)$ & $267585(16.8)$ & \\
\hline 49-53 & $434620(14.3)$ & $192594(14.8)$ & 240801 (15.1) & \\
\hline $54-58$ & $381312(12.6)$ & $173572(13.3)$ & $219277(13.8)$ & \\
\hline $59-63$ & $297621(9.8)$ & $146422(11.2)$ & 185724 (11.7) & \\
\hline \multicolumn{4}{|l|}{ Screening history: } & \multirow{6}{*}{$<0.001$} \\
\hline No history§ & $396174(13.1)$ & $167880(12.9)$ & $194251(12.2)$ & \\
\hline 1 smear $\leq 7$ years" & $446673(14.7)$ & $156727(12.0)$ & $183294(11.5)$ & \\
\hline 1 smear $>7$ years ${ }^{\star *}$ & $35164(1.2)$ & $15388(1.2)$ & $20003(1.3)$ & \\
\hline 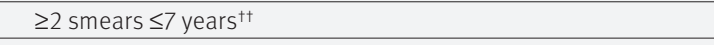 & $2095417(69.2)$ & $941575(72.2)$ & $1164713(73.2)$ & \\
\hline$\geq 2$ smears $>7$ years $\neq \neq$ & $55437(1.8)$ & $22247(1.7)$ & 29531 (1.9) & \\
\hline \multicolumn{4}{|l|}{ Socioeconomic status: } & \multirow{5}{*}{$<0.001$} \\
\hline Low & $248097(8.2)$ & $153494(11.8)$ & $108492(6.8)$ & \\
\hline Middle & $2501696(82.6)$ & $1038602(79.7)$ & $1337521(84.0)$ & \\
\hline High & $232658(7.7)$ & $87193(6.7)$ & $132863(8.3)$ & \\
\hline Unknown & $46414(1.5)$ & $24528(1.9)$ & $12916(0.8)$ & \\
\hline \multicolumn{5}{|c|}{ 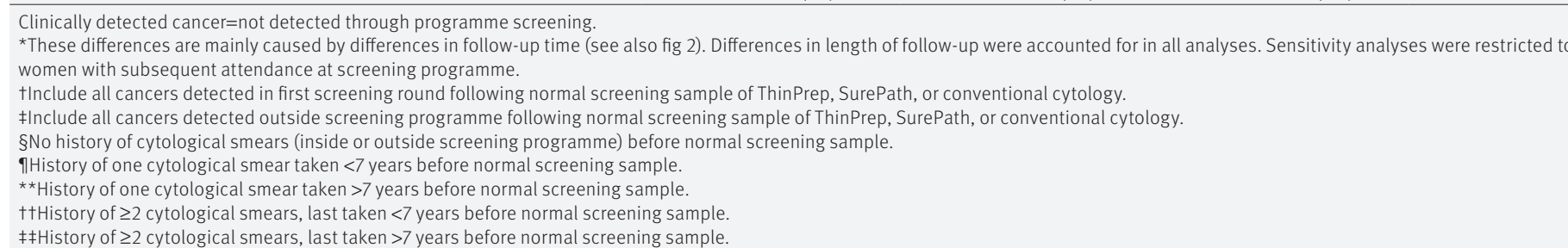 } \\
\hline
\end{tabular}

Distribution of potential confounding factors

The distribution of calendar time differed significantly between the methods of cytology testing $(\mathrm{P}<0.001)$. In $200094 \%$ of the included normal screening samples consisted of conventional cytology, whereas by 2012 this percentage had dropped to 2\% (fig 2). We also observed a large significant difference for the distributions of follow-up time. For instance, almost $80 \%$ of the normal conventional cytology samples had a follow-up time of at least 48 months, whereas for SurePath and ThinPrep this was the case for slightly more than $50 \%$ and $35 \%$ of the normal samples, respectively. Small but significant differences were also present in the distributions of socioeconomic status, screening history, and age (table 1). Missing values were imputed for socioeconomic status $(1.4 \%$ of the primary normal samples had a missing value).
Cox regression analyses of invasive cervical cancers When we compared SurePath with conventional cytology, the hazard of an invasive cancer was significantly lower (hazard ratio $0.81,95 \%$ confidence interval 0.66 to 0.99) (table 2). This decreased hazard was mainly caused by a decreased hazard of a clinically detected cancer (that is, not detected through programme screening) (hazard ratio 0.73, 0.57 to 0.93); the hazard of a screen detected cancer was similar to that of conventional cytology (0.95, 0.72 to 1.27$)$.

When we compared ThinPrep with conventional cytology, the hazard of an invasive cancer was on average non-significantly higher (hazard ratio 1.15, 0.95 to 1.38). This effect seemed to differ over time $(\mathrm{P}=0.063)$, with a hazard ratio of 0.95 ( 0.75 to 1.22$)$ in the first 44 months after the normal screening smear and of 1.40 (1.07 to 1.83) thereafter. This overall increased hazard was caused by an increased hazard of a screen detected 


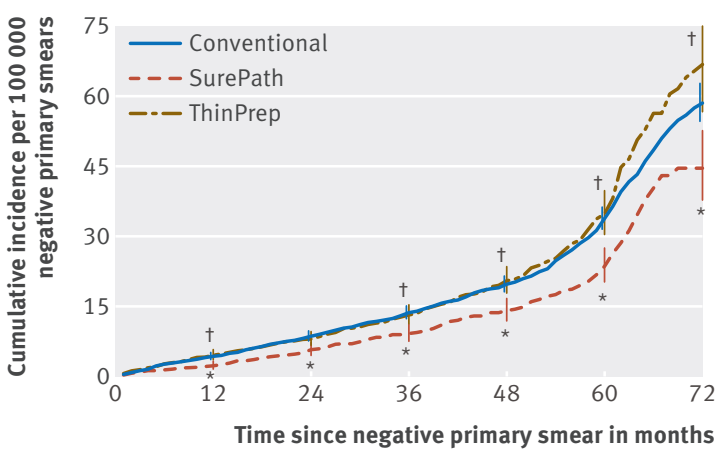

Fig 1 | Cumulative cervical cancer incidence after 100000 normal conventional cytology, SurePath, and ThinPrep samples taken within Dutch cervical cancer screening programme. Cumulative cervical cancer incidence was calculated by Kaplan-Meier analyses. $95 \%$ confidence intervals are depicted by vertical lines. *Significant difference $(P<0.05)$ between SurePath and conventional cytology. +Significant difference between SurePath and ThinPrep. No significant differences between ThinPrep and conventional cytology were detected

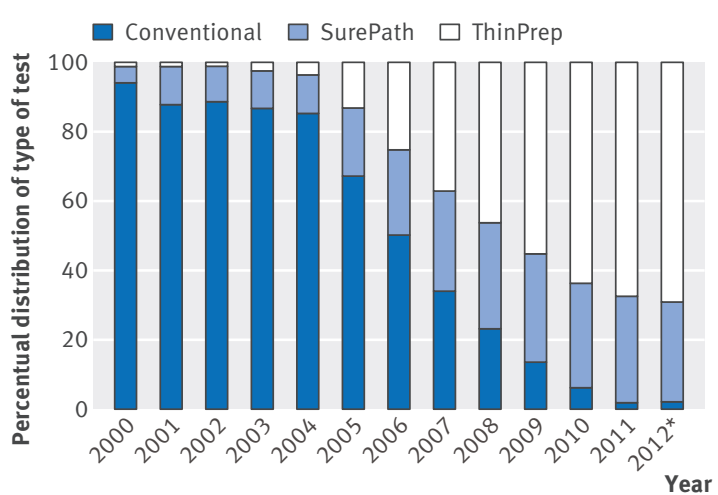

Fig 2 | Annual distribution of type of cytology used in normal screening samples taken within Dutch cervical cancer screening programme. *Until 31 March 2012. All normal screening samples taken within this time period (January 2000-March 2012) were included in study, except if type of cytology test was unknown

cancer (hazard ratio 1.56, 1.17 to 2.08), whereas the hazard of a clinically detected cancer was unaffected ( 0.96 , 0.76 to 1.20$)$.

When we compared SurePath with ThinPrep, the hazard of an invasive cancer was significantly lower (hazard ratio $0.71,0.58$ to 0.87 ). This decreased hazard was caused by both a decreased hazard of a clinically detected cancer (hazard ratio $0.76,0.59$ to 0.97 ) and a decreased hazard of a screen detected cancer (0.61, 0.46 to 0.81 ).

\section{Sensitivity analyses}

When selecting only women with at least one smear before the normal screening sample, we found that the hazard ratios were consistent with the main analyses (not restricted to women with a screening history), although the effect of ThinPrep versus conventional cytology seemed to be somewhat less pronounced (table 2). When selecting only women who attended programme screening within 72 months after a normal screening test result, we found that the hazard of a screen detected cancer increased slightly for both SurePath and ThinPrep versus conventional cytology. The hazard of a screen detected cancer for SurePath versus ThinPrep remained similar to the main analyses.

Of those women with conventional cytology at baseline followed by a subsequent screening round, 36\% were re-screened with conventional cytology, 20\% with SurePath, and 37\% with ThinPrep. For the remaining $7 \%$, the type of cytology test at re-screening was unknown. Of those with SurePath at baseline, 52\% were re-screened with SurePath, 21\% with ThinPrep, $4 \%$ with conventional cytology, and $23 \%$ with an unknown type of test. Of those with ThinPrep at baseline, 55\% were re-screened with ThinPrep, 6\% with SurePath, 3\% with conventional cytology, and $36 \%$ with an unknown type of test. The addition of the test method in the subsequent screening round as a confounding factor resulted in hazard ratios similar to the ones in the second sensitivity analysis (without this extra confounder).

\section{Difference in CIN detection rates and 72 month cumulative invasive cervical cancer incidence}

The difference in the detection of $\mathrm{CIN} \mathrm{II}^{+}$for SurePath and conventional cytology, as observed previously, ${ }^{19}$ was consistent with the observed difference in cumulative incidence of cervical cancer after a normal screening sample. The use of SurePath as primary test method resulted in 94.4 (95\% confidence interval 68.9 to 120.6) extra CIN diagnoses per 100000 screening samples, whereas the 72 month cumulative incidence of cervical cancer decreased by 11.9 ( -15.6 to -4.2$)$ (table 3$)$. The use of ThinPrep versus conventional cytology showed quite different results. Whereas the number of CIN diagnoses was similar to that with conventional cytology, the 72 month cumulative incidence of cervical cancer increased by 8.5 ( -0.7 to 18.8 ) after 100000 normal screening test results.

\section{Discussion}

The risk of invasive cervical cancer was 19\% lower after a normal SurePath sample than after a normal conventional cytological sample, which was mainly caused by a $27 \%$ lower risk of a clinically detected cancer (that is, not detected through programme screening). The use of SurePath resulted in 12 fewer cervical cancers per 100000 normal screening samples, whereas the number of detected CIN lesions increased by 94 . The risk of invasive cervical cancer seemed to be $15 \%$ higher for ThinPrep in comparison to conventional cytology, but the magnitude of the difference seemed to differ over time. Within the first 44 months after the normal screening sample, the risks were comparable; thereafter, the risk was $40 \%$ higher when using ThinPrep. Both the increased risk and the difference over time were mainly due to a $56 \%$ higher risk for a screen detected cancer. The use of ThinPrep resulted in eight additional cervical cancers per 100000 normal screening samples, whereas the number of detected CIN lesions was slightly but not statistically significantly lower. 


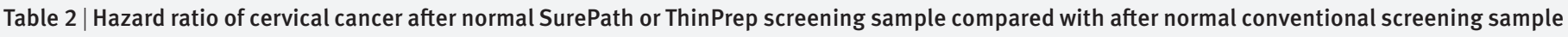
and compared with each other, overall and stratified by reason for cervical examination that led to cancer diagnosis

\begin{tabular}{|c|c|c|c|c|c|}
\hline & \multicolumn{2}{|l|}{ Main analyses } & \multicolumn{3}{|c|}{ Sensitivity analyses: adjusted HR $(95 \% \mathrm{CI})$} \\
\hline & $\begin{array}{l}\text { Unadjusted HR } \\
(95 \% \mathrm{CI})\end{array}$ & $\begin{array}{l}\text { Adjusted HR } \\
(95 \% \mathrm{CI})\end{array}$ & $\begin{array}{l}\text { (1) Restricted to } \\
\text { women with } \geq 1 \\
\text { previous smear }\end{array}$ & $\begin{array}{l}\text { (2) Restricted to } \\
\text { women attending } \\
\text { programme screening }\end{array}$ & $\begin{array}{l}\text { (3) As for (2)+ test method } \\
\text { in subsequent screening } \\
\text { round as confounding factor }\end{array}$ \\
\hline \multicolumn{6}{|l|}{ All cancers } \\
\hline SurePath $v$ CC & 0.74 (0.64 to 0.85$)$ & $0.81(0.66 \text { to } 0.99)^{\star}$ & 0.80 (0.64 to 0.99$)$ & NA & NA \\
\hline ThinPrep $v$ CC & 1.07 (0.95 to 1.22$)$ & $1.15(0.95 \text { to } 1.38)^{\dagger}$ & 1.09 (0.89 to 1.34$)$ & NA & NA \\
\hline SurePath $v$ ThinPrep & $0.69(0.58$ to 0.81$)$ & $0.71(0.58 \text { to } 0.87)^{\ddagger}$ & 0.73 (0.58 to 0.91$)$ & NA & NA \\
\hline \multicolumn{6}{|c|}{ Clinically detected cancers } \\
\hline SurePath $v$ CC & $0.70(0.59$ to 0.84$)$ & $0.73(0.57 \text { to } 0.93)^{\S}$ & $0.71(0.54$ to 0.92$)$ & NA & NA \\
\hline ThinPrep $v$ CC & $1.03(0.88$ to 1.20$)$ & $0.96(0.76 \text { to } 1.20)^{\uparrow}$ & $0.91(0.71$ to 1.16$)$ & NA & NA \\
\hline SurePath $v$ ThinPrep & $0.68(0.55$ to 0.84$)$ & $0.76(0.59 \text { to } 0.97)^{\star \star}$ & $0.78(0.60$ to 1.02$)$ & NA & NA \\
\hline \multicolumn{6}{|c|}{ Screen detected cancers } \\
\hline SurePath $v C C$ & $0.80(0.64$ to 1.02$)$ & $0.95(0.72 \text { to } 1.27)^{+\dagger}$ & 0.94 (0.68 to 1.29$)$ & $1.01(0.72$ to 1.41$)$ & $1.02(0.71$ to 1.47$)$ \\
\hline ThinPrep $v$ CC & $1.16(0.93$ to 1.44$)$ & $1.56(1.17 \text { to } 2.08)^{\dagger \dagger}$ & 1.58 (1.15 to 2.16$)$ & 1.65 (1.18 to 2.28$)$ & 1.62 (1.15 to 2.29$)$ \\
\hline SurePath $v$ ThinPrep & $0.70(0.52$ to 0.93$)$ & $0.61(0.46 \text { to } 0.81)^{t \dagger}$ & $0.59(0.43$ to 0.81$)$ & $0.61(0.44$ to 0.86$)$ & $0.63(0.43$ to 0.90$)$ \\
\hline
\end{tabular}

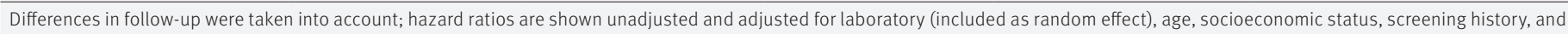
calendar time.

$\mathrm{CC}=$ conventional cytology; $\mathrm{HR}=$ hazard ratio; $\mathrm{NA}=$ not applicable.

*HR was not time dependent; $\mathrm{P}=0.559$.

tHR seemed to be time dependent, although not significantly; $P=0.063$.

FHR was not time dependent; $P=0.667$.

$\S$ HR was not time dependent; $P=0.658$

१HR was not time dependent; $P=0.306$

**HR was not time dependent; $P=0.954$

††Time dependencies not calculated for screen detected cancer, as they occur only approximately 60 months after baseline.

\section{Strengths and limitations of study}

This study is the first to compare rates of invasive cervical cancer detected after a normal screening sample between two different types of liquid based cytology tests and conventional cytology, a widely accepted proxy for examining differences in the sensitivity to detect progressive CIN lesions. In addition, we examined the drawbacks of implementation of liquid based cytology by comparing indicators of over-diagnosis.

Our study has some limitations. Firstly, we were not able to correct for the use of automated reading, although the possible influence would be small given that automated reading has been introduced in relatively few Dutch laboratories. Moreover, multiple studies have shown that $\mathrm{CIN} \mathrm{II}^{+}$detection was unaffected or slightly decreased by adding automated assisted reading to ThinPrep or SurePath. ${ }^{32-34}$ Secondly, as we did not have a unique identification code (the identification code was based on the first eight letters of the (maiden) family name and birth date), tests belonging to different women may have been allocated to a single woman (so-called fusions). However, we think it unlikely that these fusions would be correlated with the type of cytology test used. Thirdly, we did not have individual data on which type of primary test was used. Therefore, we used the date of the primary cytological smear and laboratory's conversion date fixed to the first date of the quarter to deduce which type of cytology test was used. This means that normal screening samples taken during this quarter may have been misclassified to some extent, leading to a slight underestimation of the effects. Fourthly, we were not able to censor follow-up for death and migration. However, as both mortality and migration rates are relatively low at screening ages, ${ }^{35} 36$ we do not expect that this has biased our results. Fifthly, restricting our analyses to squamous cell carcinomas, adenocarcinomas, and/or micro-invasive and macro-invasive carcinomas was not possible,

Table 3 | CIN detection rates per 100000 screening samples and 72 month cumulative cervical cancer incidence after 100000 normal screening samples for conventional cytology, and difference in those measures for SurePath and ThinPrep compared with conventional cytology

\begin{tabular}{|c|c|c|c|}
\hline & $\begin{array}{l}\text { Conventional } \\
\text { cytology }\end{array}$ & $\begin{array}{l}\text { SurePath } v \\
\text { conventional } \\
\text { cytology }\end{array}$ & $\begin{array}{l}\text { ThinPrep } v \\
\text { conventional cytology }\end{array}$ \\
\hline \multicolumn{4}{|c|}{ No of CIN diagnoses per 100000 screening samples } \\
\hline CIN I & 216.1 & 30.1 (18.1 to 42.8$)$ & $-3.5(-14.3$ to 7.9$)$ \\
\hline CIN II & 220.0 & $31.2(19.0$ to 44.1$)$ & $9.4(-2.1$ to 21.5$)$ \\
\hline CIN III & 495.0 & $30.3(12.0$ to 49.3$)$ & $-12.2(-29.6$ to 5.9$)$ \\
\hline Total CIN & 931.0 & $94.4(68.9$ to 120.6$)$ & $-6.8(-30.6$ to 17.6$)$ \\
\hline \multicolumn{4}{|c|}{ No of cervical cancer diagnoses after 100000 normal screening samples ${ }^{\star}$} \\
\hline Total cancers & $58.5(54.6$ to 62.7$)$ & $-11.9(-15.6$ to -4.2$)$ & $+8.5(-0.7$ to 18.8$)$ \\
\hline
\end{tabular}

Numbers were corrected for confounding factors. $95 \%$ confidence intervals are given in parentheses.

$\mathrm{CIN}=$ cervical intraepithelial neoplasia.

*Differences in distribution of follow-up were taken into account, and laboratories were included as random effects in model. 
as this information in PALGA is not accurate and many values are missing. Finally, we did not correct for possible learning curve effects, as the aim of our study was to examine the effect of using SurePath and ThinPrep in routine practice, which also includes a possible learning effect.

\section{Effect of confounding factors and sensitivity analyses}

As laboratory and calendar time were clearly correlated with the moment of implementation of liquid based cytology, their confounding effects were much more pronounced than those of age, screening history, and socioeconomic status. Although significantly different, their distribution differences were very small and, therefore, confounding effects were negligible. It is possible that we did not take into account the effect of other (unknown) potential confounders. The fact that we found no large differences in age, screening history, and socioeconomic status is reassuring, however.

We found that the effect of ThinPrep compared with conventional cytology seemed to be somewhat less pronounced in women with a screening history compared with our main analysis (also including women without a screening history). This suggests that the risk of a cervical cancer after a normal ThinPrep screening sample is perhaps increased more in women without versus with a screening history.

Restricting the analysis to women who attended the next screening round slightly increased the risk of a screen detected cancer for both SurePath and ThinPrep, although the difference between these two types of liquid based cytology test remained similar. This may have resulted in a slight underestimation of the hazard ratios between SurePath and conventional cytology and between ThinPrep and conventional cytology.

In an ideal situation, the type of cytology test used would differ between the groups only at baseline to ensure that results are not biased by differences in sensitivity to detect a cervical cancer in the episode following the normal screening sample. Our finding that the addition of the second type of test (in the subsequent episode) as confounder did not change our results was reassuring.

\section{Explanation of main results}

In our previous study, using the same data as in this study, we showed that the detection of $\mathrm{CIN} \mathrm{II}^{+}$was increased by using SurePath compared with conventional cytology, whereas it was unaffected by using ThinPrep. ${ }^{19}$ As the use of SurePath resulted in decreased rates of cervical cancer after a normal screening sample, this indicates that at least part of the extra detected CIN lesions were progressive. As the use of ThinPrep seemed to result in increased cancer rates, this suggests that fewer of the detected CIN lesions were progressive.

In addition, we showed that the use of SurePath was associated with lower rates of cervical cancer after a normal screening sample compared with the use of ThinPrep, indicating that the sensitivity to detect progressive CIN lesions is higher for SurePath. These suggested differences in sensitivity are most likely caused by differences between the techniques of the liquid based cytology tests, such as the extent of fixation, the technique of taking a representative sample from the vial, and the retention of the brush (the collecting device) in the fluid. ${ }^{3738}$ Studies have shown that retaining the brush, as is done when using SurePath, is associated with an increased cell yield compared with rinsing and discarding the brush, as is done when using ThinPrep. ${ }^{39} 40$

\section{Extrapolation of results}

The cumulative incidence of cancer after a normal sample seemed to be higher for ThinPrep than for conventional cytology mainly because the risk of a screen detected cancer after a normal sample was higher. In general, screen detected cancers are found at a lower stage than clinically diagnosed ones, so their survival rate is probably better. ${ }^{41}$ Therefore, the suggested negative effect of using ThinPrep is probably less pronounced for cervical cancer mortality than it is for incidence. The opposite is true for the positive effect of SurePath, as we found that SurePath was primarily protective for clinically detected cancers. As no data on mortality were available, we were not able to estimate the effects of implementation of liquid based cytology on cervical cancer mortality. Although our results may not seem very relevant for the future of the Dutch cervical cancer screening programme, as primary cytology screening has recently been replaced by primary human papillomavirus screening, they will certainly be relevant to other countries with organised primary cytology screening programmes that have switched to using SurePath and/or ThinPrep or will switch in the near future.

\section{Over-diagnosis}

An important drawback of cervical cancer screening is over-diagnosis and over-treatment of CIN lesions (that is, of lesions that would never have progressed to clinical cervical cancer in the absence of screening). The possible increase in such over-diagnosis and over-treatment related to more sensitive screening should be taken into account when considering new screening options. With SurePath, the prevention of 12 extra cervical cancers within the first six years after screening was accompanied by the detection of 94 extra CIN lesions at that screening round. Most of these CIN lesions would never have become invasive cancer and could therefore be classified as over-diagnosis. Assuming that only CIN II+ are treated, replacing conventional cytology with SurePath would have implied that roughly five more CIN treatments are performed to prevent one additional cervical cancer diagnosis. However, in a subsequent screening round for the same cohort, increased detection rates tend to wane, and so do over-diagnosis and over-treatment. This effect was also observed in randomised controlled trials in which cytology screening was replaced by highly sensitive human papillomavirus based screening in the intervention arm. The detection of high grade CIN was increased at the prevalence 
round but decreased at the subsequent incidence round, accompanied by a reduced risk of interval cancers. ${ }^{4243}$ Therefore, in our study, the number of additional CIN treatments per additionally prevented cancer is, if anything, overestimated.

\section{Comparison with literature}

Whether one cytology test is preferred over another should depend not only on the sensitivity to detect progressive CIN lesions and rates of over-diagnosis but also on factors such as the possibility to test for the presence of human papillomavirus in the residual material and the percentage of unsatisfactory smears. Fontaine et al have shown that unsatisfactory rates are significantly lower when using SurePath instead of ThinPrep..$^{44}$ Although this was not shown in our previous study, ${ }^{19}$ we then found similar results (an odds ratio of $0.74,0.72$ to 0.75 ) when comparing unsatisfactory rates between SurePath and ThinPrep.

\section{Conclusions}

The six year cumulative incidence of cervical cancer after a normal screening sample was significantly lower for SurePath than for conventional cytology and ThinPrep, strongly suggesting that the sensitivity of SurePath to detect progressive CIN lesions is higher. The use of ThinPrep compared with the use of conventional cytology seemed to be associated with a higher cumulative cancer incidence, suggesting that the sensitivity to detect progressive CIN lesions is lower, although results were non-significant. Our findings should provoke reconsideration of the assumed similarity in the sensitivity for progressive CIN between the different types of liquid based cytology tests and conventional cytology.

We thank the regional coordinating pathologists J C van der Linden, J E Boers, J Bulten, and M Kliffen for their help in collecting the data. We thank A G Siebers for linking data from the laboratories to the PALGA database.

Contributors: LIHO linked laboratories' data to the PALGA database. KR designed the study, did the analyses, and wrote the manuscript. CWNL gave statistical advice. All authors interpreted the results, reviewed the paper critically, and approved the final version submitted. MvB is the guarantor.

Funding: This is an independent report commissioned and funded by the Dutch National Institute for Public Health and the Environment (RIVM, 007/12 V\&Z NvdV/EM). The funding source (a non-profit organisation) had no involvement in the study design, data collection, data analysis, interpretation of the data, writing of the report, or the decision to submit the paper for publication.

Competing interests: All authors have completed the ICMJE uniform disclosure form at www.icmje.org/coi_disclosure.pdf (available on request from the corresponding author) and declare: KR, SKN, CP, IMCMdK, and MvB received funding from the Dutch National Institute for Public Health and the Environment (RIVM, 007/12 V\&Z NvdV/EM); KR was invited by Becton Dickinson to present this work at the European Congress of Cytology in Geneve, although she did not receive any salary, compensation, or bonus; MR was involved in comparative studies of new generation human papillomavirus tests, for which Roche, Genomica, Qiagen, Hologic/Gen-Probe, BD, and Rovers provided assays and instrumentations; MR did not receive any salary, compensation, or bonuses for work on any of the projects from any of the companies and does not hold companies' stock; she and her (former) employer received speaker fees from Qiagen; no other relationships or activities that could appear to have influenced the submitted work.

Ethical approval: Data retrieval was approved by the board of PALGA, the nationwide network and registry of histo- and cytopathology in the Netherlands.
Data sharing: Individual level data can be requested from the nationwide network and registry of histo- and cytopathology in the Netherlands (PALGA).

Transparency statement: The lead author (the manuscript's guarantor) affirms that the manuscript is an honest, accurate, and transparent account of the study being reported; that no important aspects of the study have been omitted; and that any discrepancies from the study as planned have been explained

This is an Open Access article distributed in accordance with the Creative Commons Attribution Non Commercial (CC BY-NC 4.0) license, which permits others to distribute, remix, adapt, build upon this work non-commercially, and license their derivative works on different terms, provided the original work is properly cited and the use is non-commercial. See: http://creativecommons.org/licenses/ by-nc/4.0/.

1 Rask J, Lynge E, Franzmann M, et al. Impact of technology on cytology outcome in cervical cancer screening of young and older women Int / Cancer 2014:134:2168-79 doi:101002/ijc 28532

2 Public Health England. Cervical screening: a pocket guide.NHS Cervical Cancer Screening Programme, 2009.

3 Cox JT. History of the use of HPV testing in cervical screening and in the management of abnormal cervical screening results. I Clin Viro 2009;45(Suppl 1):S3-12. doi:10.1016/S1386-6532(09)70002-2.

4 Albrow R, Kitchener H, Gupta N, Desai M. Cervical screening in England: the past, present, and future. Cancer Cytopathol 2012:120:87-96. doi:10.1002/cncy.20203.

5 Colgan TJ, McLachlin CM, Cotterchio M, Howlett R, Seidenfeld AM, Ma VM. Results of the implementation of liquid-based cytology-SurePath in the Ontario screening program. Cancer 2004:102:362-7. doi:10.1002/cncr.20656.

6 Fremont-Smith M, Marino J, Griffin B, Spencer L, Bolick D. Comparison of the SurePath liquid-based Papanicolaou smear with the conventional Papanicolaou smear in a multisite direct-to-vial study. Cancer 2004;102:269-79. doi:10.1002/cncr.20599.

7 Beerman H, van Dorst EB, Kuenen-Boumeester V, Hogendoorn PC. Superior performance of liquid-based versus conventional cytology in a population-based cervical cancer screening program. Gynecol Oncol 2009;112:572-6. doi:10.1016/j.ygyno.2008.12.012.

8 Siebers AG, Klinkhamer PJ, Arbyn M, Raifu AO, Massuger LF, Bulten J. Cytologic detection of cervical abnormalities using liquid-based compared with conventional cytology: a randomized controlled trial. Obstet Gynecol 2008;112:1327-34. doi:10.1097/ AOG.Ob013e31818c2b20.

9 Sigurdsson K. Is a liquid-based cytology more sensitive than a conventional Pap smear?Cytopathology 2013;24:254-63. doi:10.1111/cyt.12037.

10 Arbyn M, Bergeron C, Klinkhamer P, Martin-Hirsch P, Siebers AG, Bulten J. Liquid compared with conventional cervical cytology: a systematic review and meta-analysis. Obstet Gynecol 2008;111:16777. doi:10.1097/01.AOG.0000296488.85807.b3.

11 Whitlock EP, Vesco KK, Eder M, Lin JS, Senger CA, Burda BU. Liquid-based cytology and human papillomavirus testing to screen for cervical cancer: a systematic review for the U.S. Preventive Services Task Force. Ann Intern Med 2011;155:687-97, W214-5. doi:10.7326/0003-4819-155-10-201111150-00376.

12 Coste J, Cochand-Priollet B, de Cremoux P, et al. French Society of Clinical Cytology Study Group. Cross sectional study of conventional cervical smear, monolayer cytology, and human papillomavirus DNA testing for cervical cancer screening. BMJ 2003;326:733. doi:10.1136/ bmi.326.7392.733.

13 Hussein T, Desai M, Tomlinson A, Kitchener HC. The comparative diagnostic accuracy of conventional and liquid-based cytology in a colposcopic setting. BJOG 2005;112:1542-6. doi:10.1111/j.1471-0528.2005.00699.x.

14 Ronco G, Cuzick J, Pierotti P, et al. Accuracy of liquid based versus conventional cytology: overall results of new technologies for cervical cancer screening. randomised controlled trial. BM/ 2007:335:28. doi:10.1136/bmj.39196.740995.BE.

15 Siebers AG, Klinkhamer PJ, Grefte JM, et al. Comparison of liquid-based cytology with conventional cytology for detection of cervical cancer precursors: a randomized controlled trial. JAMA 2009;302:1757-64. doi:10.1001/jama.2009.1569.

16 Taylor S, Kuhn L, Dupree W, Denny L, De Souza M, Wright TC Jr. Direct comparison of liquid-based and conventional cytology in a South African screening trial. Int J Cancer 2006;118:957-62. doi:10.1002/ ijc.21434.

17 Ferenczy A, Robitaille J, Franco E, Arseneau J, Richart RM, Wright TC Conventional cervical cytologic smears vs. ThinPrep smears. A paired comparison study on cervical cytology. Acta Cytol 1996;40:1136-42. doi:10.1159/000333971.

18 Sykes PH, Harker DY, Miller A, et al. A randomised comparison of SurePath liquid-based cytology and conventional smear cytology in a colposcopy clinic setting. BJOG 2008;115:1375-81 doi:10.1111/j.1471-0528.2008.01865.x 
19 Rozemeijer K, Penning C, Siebers AG, et al. Comparing SurePath, ThinPrep, and conventional cytology as primary test method: SurePath is associated with increased CIN II+ detection rates. Cancer Causes Control 2016;27:15-25. doi:10.1007/s10552-015-0678-1.

20 Rebolj M, Rask J, van Ballegooijen M, et al. Cervical histology after routine ThinPrep or SurePath liquid-based cytology and computerassisted reading in Denmark. BrJ Cancer 2015;113:1259-74. doi:10.1038/bjc.2015.339.

21 Rebolj M, van Ballegooijen M, van Kemenade F, Looman C, Boer R, Habbema JD. No increased risk for cervical cancer after a broader definition of a negative Pap smear. Int J Cancer 2008;123:2632-5. doi:10.1002/ijc.23803.

22 Casparie M, Tiebosch AT, Burger G, et al. Pathology databanking and biobanking in The Netherlands, a central role for PALGA, the nationwide histopathology and cytopathology data network and archive. Cell Oncol 2007;29:19-24.

23 Rebolj M. Recent developments in the Dutch cervical cancer screening programme. Erasmus University Rotterdam, 2008

24 Blok M. [Description of the Paleba system and its algorithms 2012]. [Dutch]. CACl, by order of Palga, 2013.

25 Dutch Society for Pathology. [Practice guideline version 3.2 - For quality assurance of cytopathological research of the cervix], Dutch. Soc Pathol 2012.

26 The Netherlands Institute for Social Research. [From high to low; from low to high - The social-spatial development of quarters between 1971 and 1995]. Den Haag, 1998.

27 Vesco KK, Whitlock EP, Eder M, Burda BU, Senger CA, Lutz K. Risk factors and other epidemiologic considerations for cervical cancer screening: a narrative review for the U.S. Preventive Services Task Force. Ann Intern Med 2011;155:698-705, W216. doi:10.7326/0003-4819-155-10-201111150-00377.

28 Rozemeijer K, van Kemenade FJ, Penning C, et al. Exploring the trend of increased cervical intraepithelial neoplasia detection rates in the Netherlands. / Med Screen 2015;22:144-50. doi:10.1177/0969141315580836.

29 Froment MA, Gomez SL, Roux A, DeRouen MC, Kidd EA. Impact of socioeconomic status and ethnic enclave on cervical cancer incidence among Hispanics and Asians in California. Gynecol Oncol 2014;133:409-15. doi:10.1016/j.ygyno.2014.03.559.

30 Jensen KE, Hannibal CG, Nielsen A, et al. Social inequality and incidence of and survival from cancer of the female genital organs in a population-based study in Denmark, 1994-2003. Eur J Cancer 2008;44:2003-17. doi:10.1016/j.ejca.2008.06.014.

31 Harrell F. Regression modeling strategies - with applications to linear models, logistic regression, and survival analysis.1st ed. SpringerVerlag New York, 2001.

32 Klug SJ, Neis KJ, Harlfinger W, et al. A randomized trial comparing conventional cytology to liquid-based cytology and computer assistance. Int J Cancer 2013;132:2849-57. doi:10.1002/ijc.27955.
33 Palmer TJ, Nicoll SM, McKean ME, et al. Prospective parallel randomized trial of the MultiCyte ${ }^{\mathrm{TM}}$ ThinPrep $(\mathbb{R})$ imaging system: the Scottish experience. Cytopathology 2013;24:235-45. doi:10.1111/j.1365-2303.2012.00982.x.

34 Kitchener HC, Blanks R, Dunn G, et al. Automation-assisted versus manual reading of cervical cytology (MAVARIC): a randomised controlled trial. Lancet Oncol 2011;12:56-64. doi:10.1016/ S1470-2045(10)70264-3

35 Mortality: What is the current situation? National Institute of Public Health and the Environment, 2016 (available from http://www. nationaalkompas.nl/bevolking/migratie/huidig/).

36 Migration: What is the current situation? National Institute of Public Health and the Environment, 2016 (available from http://www. nationaalkompas.nl/bevolking/migratie/huidig/).

37 Zhao FH, Hu SY, Bian JJ, et al. Comparison of ThinPrep and SurePath liquid-based cytology and subsequent human papillomavirus DNA testing in China. Cancer Cytopathol 2011:119:387-94. doi:10.1002/ cncy.20177.

38 Thiryayi SA, Marshall J, Rana DN. An audit of liquid-based cervical cytology screening samples (ThinPrep and SurePath) reported as glandular neoplasia. Cytopathology 2010;21:223-8 doi:10.1111/j.1365-2303.2009.00695.x.

39 Bigras G, Rieder MA, Lambercy JM, et al. Keeping collecting device in liquid medium is mandatory to ensure optimized liquid-based cervical cytologic sampling. J Low Genit Tract Dis 2003;7:168-74. doi:10.1097/00128360-200307000-00003.

40 Umana A, Dunsmore H, Herbert A, Jokhan A, Kubba A. Are significant numbers of abnormal cells lost on the discarded ThinPrep $®$ broom when used for cervical cytology?Cytopathology 2013;24:228-34. doi:10.1111/cyt.12029.

41 van der Aa MA, Schutter EM, Looijen-Salamon M, Martens JE, Siesling $S$. Differences in screening history, tumour characteristics and survival between women with screen-detected versus not screen-detected cervical cancer in the east of The Netherlands, 1992-2001. Eur I Obstet Gynecol Reprod Biol 2008;139:204-9. doi:10.1016/j. ejogrb.2007.10.017.

42 Arbyn M, Ronco G, Anttila A, et al. Evidence regarding human papillomavirus testing in secondary prevention of cervical cancer. Vaccine 2012;30(Suppl 5):F88-99. doi:10.1016/j. vaccine.2012.06.095.

43 Ronco G, Dillner J, Elfström KM, et al. International HPV screening working group. Efficacy of HPV-based screening for prevention of invasive cervical cancer: follow-up of four European randomised controlled trials. Lancet 2014:383:524-32. doi:10.1016/ S0140-6736(13)62218-7.

44 Fontaine D, Narine N, Naugler C. Unsatisfactory rates vary between cervical cytology samples prepared using ThinPrep and SurePath platforms: a review and meta-analysis. BMJ Open 2012;2:e000847. doi:10.1136/bmjopen-2012-000847.

\section{Supplementary materials}

